\title{
Quantum atomic delocalization vs. structural disorder in amorphous silicon
}

\author{
Carlos P. Herrero \\ Instituto de Ciencia de Materiales, C.S.I.C., Campus de Cantoblanco, 28049 Madrid, Spain \\ and \\ Institut für Theoretische Festkörperphysik, Universität Karlsruhe, D-76128 Karlsruhe, Germany
}

(July 7, 2017)

\begin{abstract}
Quantum effects on the atom delocalization in amorphous silicon have been studied by path-integral Monte Carlo simulations from 30 to $800 \mathrm{~K}$. The quantum delocalization is appreciable vs. topological disorder, as seen from structural observables such as the radial distribution function (RDF). At low temperatures, the width of the first peak in the RDF increases by a factor of 1.5 due to quantum effects. The overall anharmonicity of the solid vibrations at finite temperatures in amorphous silicon is clearly larger than in the crystalline material. Low-energy vibrational modes are mainly located on coordination defects in the amorphous material.
\end{abstract}

Structural and dynamical aspects of amorphous solids have been studied for many years by several experimental and theoretical techniques [1.2]. In spite of the intense work devoted to determine the structure of amorphous materials at an atomic level, there are still many open questions concerning the short and intermediate length scales in these materials [3]. In this context, amorphous silicon, apart from its technological interest, is a model system to analyze energy-minimized structures in glassy solids [4], as well as low-energy excitations in disordered semiconductors [5].

In the last decade, computer simulations of semiconductors with topological disorder have been performed, in order to obtain insight into different structural and dynamical properties of these materials. Early simulations [6] of amorphous silicon (a-Si) were based on the continuous random network model (see ref. [1]), and later, most simulations of this material have been carried out by molecular dynamics (MD) with empirical interatomic potentials [7 10]. Ab initio MD simulations with the Car-Parrinello method were also carried out for a-Si, and their results for the atomic structure, the phonon spectrum, and the electronic properties were in good agreement with experimental data [11. These simulations, however, treat the silicon nuclei as classical particles, thus neglecting typical quantum effects such as tunneling or zero-point motion. The quantum delocalization of the atomic nuclei in solids is usually taken into account through a harmonic approximation, but such an approach is in principle not valid for amorphous solids, due to the presence of highly anharmonic low-energy vibrations in these materials 12,13]. In last years, ab initio path-integral MD simulations have become possible for small molecules, though they are still hardly feasible for detailed studies of solids [14].

An important point, when considering average structural properties of amorphous materials, is the question whether quantum effects can be important vs. struc- tural disorder. In particular, one may ask whether the radial distribution function (RDF) obtained in classical simulations will be changed by introducing quantum effects, with the consequent quantum delocalization of the atoms, which can be noticeable mainly at low temperatures. Thus, two factors appear to compete in the broadenig of the RDF peaks at low $T$ : structural disorder and quantum delocalization. As a first approach, one expects that for amorphous materials with heavy atoms (small zero-point delocalization), the structural disorder will broaden the peaks more than the zero-point motion, and the contrary will happen for disordered materials with light atoms.

The purpose of this paper is twofold: First, to analyze whether the average structural properties of amorphous silicon are affected by quantum effects, and second, to check the validity of a harmonic approximation to obtain average quantities (energy, mean atom delocalization) at finite temperatures. With these goals, amorphous silicon has been studied by path-integral (PI) Monte Carlo (MC) simulations in a temperature range from 30 to 800 $\mathrm{K}$. This computational technique is now well established as a tool to study many-body problems in which anharmonic effects can be non-negligible. It is based on the Feynman path-integral formulation of statistical mechanics, according to which the partition function of a quantum system can be evaluated through a discretization of the density matrix along cyclic paths, composed of a finite number of imaginary-time steps 157. This Trotter number, $N$, causes the appearance in the simulations of $N$ 'replicas' for each quantum particle. Such replicas are treated as classical particles, and the corresponding partition function is sampled by the Metropolis method [16]. More details on this simulation procedure can be found elsewhere [17,18].

The interatomic interaction has been modelled by the Stillinger-Weber (SW) potential, [19] which was employed in earlier PI MC simulations of crystalline sili- 


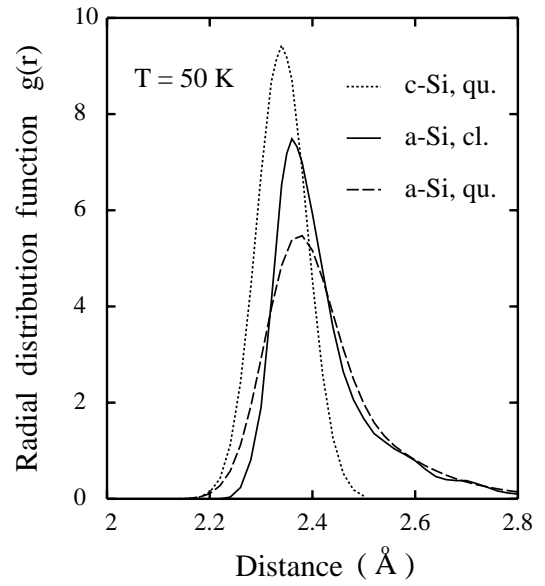

FIG. 1. Radial distribution function in the region from 2 to $2.8 \AA$, as obtained from Monte Carlo simulations of a-Si at $50 \mathrm{~K}$ : classical (continuous line) and quantum (dashed line). Results of quantum simulations for c-Si are plotted as a dotted line.

con (c-Si), giving results (total energy, quantum delocalization of the $\mathrm{Si}$ atoms) in good agreement with those derived from experiment 18. This interatomic potential was also shown to be adequate to simulate structural and dynamical properties of a-Si in classical MD simulations [7, ק]. A cubic cell for a-Si with a size length of 16.6 $\AA$, and including 216 atoms, was generated by using a simulated annealing process similar to that described in refs. [7] and [8], where an amorphous structure was generated by MD simulations, via slowly cooling from the melt. In contrast with these works, we have carried out the simulated annealing by using classical MC simulations with the same SW potential employed later for the quantum simulations. In this process, the temperature $T$ was reduced from 2400 to $200 \mathrm{~K}$ in 15 steps, involving a total of $3 \times 10^{6} \mathrm{MC}$ steps (MCS). Several simulation cells were generated by the same procedure to check that the average results obtained did not depend on the particular cell under consideration. The structural characteristics of our generated cells are similar to those found in earlier MD simulations with the SW potential, in particular the radial distribution function (RDF) and the fraction of five-fold coordinated atoms $(\sim 20 \%)$. Once the simulation cell for a-Si was generated, we carried out PI MC simulations in a temperature range from 30 to $800 \mathrm{~K}$. The Trotter number, $N$, was made temperaturedependent $(N \sim 2000 / T)$, in order to keep the numerical error caused by the discretization of the path integrals lower than $\pm 1 \mathrm{meV}$ per Si atom [18]. At each temperature, 5000 MCS were carried out for system equilibration, and 15,000 to calculate the average variables of our simulation cell. Each MCS included an update of the coordinates of all replicas for each Si nucleus, as well as an attempt to move the center-of-gravity of the cyclic path associated to each nucleus. The results of our simula-

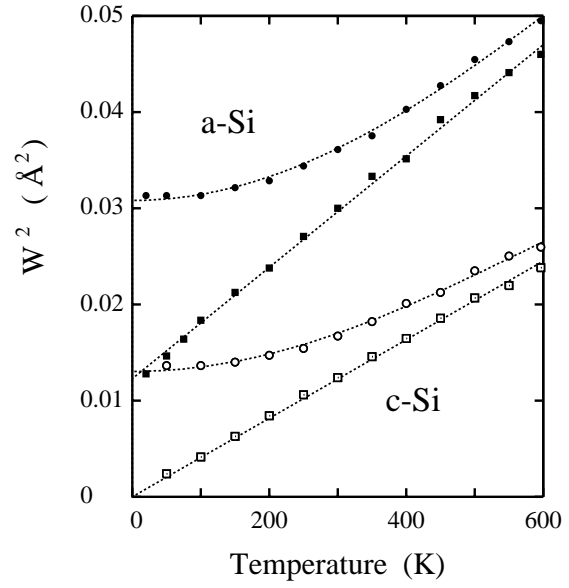

FIG. 2. Squared FWHM of the first peak in the radial distribution function, as a function of temperature, as derived from Monte Carlo simulations: open squares, classical c-Si; open circles, quantum c-Si; black squares, classical a-Si; black circles, quantum a-Si. Lines are guides to the eye.

tions are compared to those found for crystalline silicon with the same simulation method and the same number of atoms in the simulation cell. In order to find out the importance of quantum effects, classical MC simulations were also carried out for amorphous and crystalline silicon in the same temperature range.

Traditionally, the RDF has been a useful means to characterize the structure of amorphous materials. In fig. 1 we present the RDF for a-Si obtained from our classical (continuous line) and quantum (dashed line) Monte Carlo simulations at $50 \mathrm{~K}$, in the range from 2 to $2.8 \AA$. For comparison, we give also the RDF found for c-Si in quantum simulations at the same temperature (dotted line). In the classical simulations of amorphous silicon, the first peak in the RDF has its maximum at a distance $r_{m}=2.36 \AA$, close to the nearest-neighbor distance in c-Si. However, in these simulations the average $\mathrm{Si}-\mathrm{Si}$ distance in a-Si is found to be somewhat higher $(2.43 \AA)$, as a consequence of the peak asymmetry (we employ a nearest-neighbor cutoff distance of $2.9 \AA$ ). For quantum a-Si, we find the peak-maximum slightly shifted to higher distances $\left(r_{m}=2.37 \AA\right)$, but the mean Si-Si distance is the same as for 'classical' a-Si. The peak found in quantum simulations at low temperatures is, however, clearly broader than that obtained in the classical case.

In fig. 2 we show the squared full-width-at-halfmaximum (FWHM, denoted here by $W$ ), derived from our MC simulations (both, classical and quantum) for crystalline and amorphous silicon. In the classical simulations, one finds a linear dependence of $W^{2}$ vs. T. For the crystal (open squares), $W$ goes to zero at low $T$, as should happen for a classical approach. For amorphous silicon (black squares), $W$ converges to a finite value at $T$ $=0\left(W_{0}=0.11 \AA\right)$, as a consequence of structural disorder. Our value of $W$ for classical simulations of a-Si at $T$ 
$=300 \mathrm{~K}(W=0.175 \AA)$ compares well with that found in earlier MD simulations $(W=0.17 \pm 0.01 \AA$ ) [10].

The width $W$ obtained for c-Si in quantum simulations at low temperatures (open circles) is due to zero-point motion of the Si atoms in the crystal. This width is found to be similar to that obtained for a-Si in the classical simulations (the latter being due to structural disorder in the amorphous material). As an important result, we find that, at low $T, W^{2}$ for quantum a-Si (black circles) is found to be about 2.5 times the value corresponding to 'classical' a-Si. For distances larger than $3 \AA$, the RDF's obtained in classical and quantum simulations coincide. To our knowledge, there are no experimental data on aSi to compare directly with our calculated $W$ values, but the FWHM found for amorphous germanium at room temperature [20] is of the order of that obtained here.

The structural disorder present in a-Si affects the bond lenghts and bond angles as follows: Bond lengths are, in average, larger in a-Si than in c-Si, even for $\mathrm{Si}-\mathrm{Si}$ bonds between four-fold coordinated atoms. Also, the bondangle distribution for four-fold coordinated atoms in a-Si is broader than in the crystalline material. In fact, for $T=50 \mathrm{~K}$ we find a $\mathrm{Si}-\mathrm{Si}-\mathrm{Si}$ angle distribution with a FWHM of $22^{\circ}$ around the tetrahedral angle. At this temperature, the corresponding width for c-Si is $5.5^{\circ}$, as derived from our quantum simulations. This angle distribution is much broader for coordination defects in a-Si, with a constant width of $\sim 70^{\circ}$, irrespective of temperature. The longer average Si-Si distance in a-Si causes: (i) a decrease in the stretching frequency of the $\mathrm{Si}-\mathrm{Si}$ bond, in particular, and a decrease in the zero-point energy $\frac{1}{2}\langle\hbar \omega\rangle$ obtained from the vibrational density of states (VDOS); and (ii) an increase in the anharmonicity of the solid vibrations, related to the metastability of the amorphous solid. The same trend is found for the effect of angle distorsions on the zero-point vibrational energy and on the anharmonicity (see below).

An important value that can be obtained directly from the quantum simulations is the spatial delocalization of the silicon atoms in the material. One expects that this delocalization will be higher for a-Si than for c-Si due to the low-energy vibrational modes that appear in disordered materials [8,13], and that cause an increase in the VDOS at low energies, as compared with the crystalline solid. In fig. 3 we show the average delocalization, $(\Delta r)^{2}$, of the $\mathrm{Si}$ atoms vs. T. Open squares correspond to c-Si, and black squares and circles represent the delocalization of four- and five-fold coordinated $\mathrm{Si}$ atoms in amorphous silicon, respectively. The continuous and dashed lines were obtained from the VDOS obtained for the SW potential [8] by assuming a harmonic approximation. As expected, the mean-square displacement of the four-fold coordinated atoms in a-Si is clearly larger than that corresponding to c-Si. Moreover, five-fold coordinated $\mathrm{Si}$ atoms (black circles in fig. 3) are more delocalized than four-fold coordinated atoms, indicating that the former have a larger contribution to localized low-energy vibrational modes. This is in line with the fact that coordi-

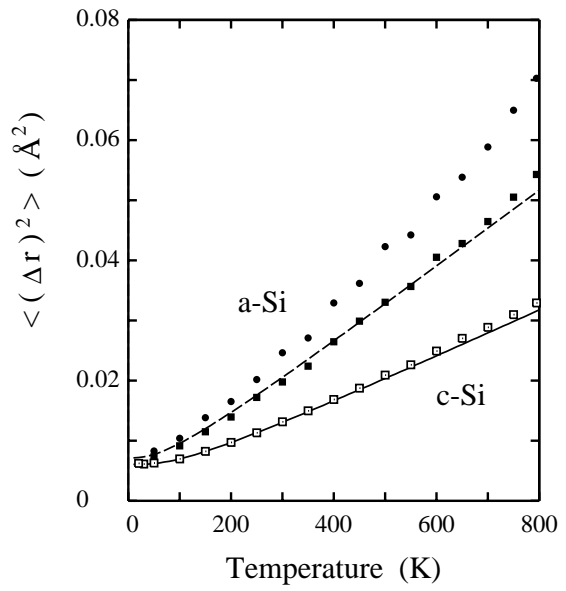

FIG. 3. Mean-square displacement of the atoms in crystalline and amorphous silicon. Open squares: c-Si; black squares and circles for four- and five-fold coordinated atoms in a-Si, respectively. The continuous and dashed lines were calculated from the vibrational density-of-states for crystalline and amorphous silicon, respectively.

nation defects give rise to extra states at low frequencies [9]. Note that a small extra contribution of low-frequency modes to the local VDOS for five-fold coordinated atoms, can have an appreciable effect on $(\Delta r)^{2}$ at finite temperatures, as that shown in fig. 3. The results obtained for the mean-square displacement of four-fold coordinated atoms follow closely (for both a-Si and c-Si) the lines derived from the harmonic approximation. Only at $T>$ $600 \mathrm{~K}$, a slight deviation of the PI MC results toward higher values is found in both cases.

The influence of quantum effects on the atomic motion can be quantified by calculating the correlations between atom displacements. We define the correlation $\rho$ for displacements of atom pairs by the quantity:

$$
\rho(r)=\frac{\left\langle\mathbf{u}_{i} \cdot \mathbf{u}_{j}\right\rangle}{\sqrt{\left\langle\mathbf{u}_{i}^{2}\right\rangle\left\langle\mathbf{u}_{j}^{2}\right\rangle}},
$$

where $\mathbf{u}_{i}\left(\mathbf{u}_{j}\right)$ is the displacement of atom $i(j)$ from its equilibrium position, and $r$ is the distance between the equilibrium positions of atoms $i$ and $j$. We have calculated this pair correlation as a function of $r$ in quantum and classical MC simulations. In fig. 4 we present the correlation $\rho(r)$ for both amorphous and crystalline silicon at $T=50$ and $600 \mathrm{~K}$. The dashed and dotted lines correspond to a-Si at these temperatures. At $T=600$ $\mathrm{K}$, we find that the correlation $\rho$ is $\sim 0.4$ at a nearestneighbor distance $r=2.35 \AA$, and decreases as the interatomic distance increases (dotted line). For $r>6 \AA$, this correlation is nearly zero. At low $T$ (dashed line), quantum delocalization causes a decrease in the correlation of atom displacements. The black and white circles were obtained from quantum simulations of crystalline silicon. These points follow closely the corresponding lines for 


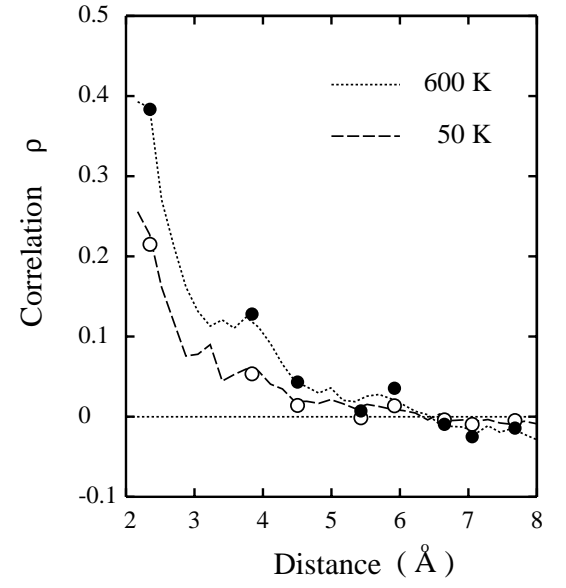

FIG. 4. Correlation $\rho$ between atom displacements vs. interatomic distance, at two different temperatures: Open circles, c-Si at $50 \mathrm{~K}$; black circles, c-Si at $600 \mathrm{~K}$; dashed line, a-Si at $50 \mathrm{~K}$; dotted line, a-Si at $600 \mathrm{~K}$. Error bars are of the order of the symbol size.

amorphous silicon. This means that, in spite of the apparent differences between the VDOS of both materials [8], the correlations between atom displacements do not depend appreciably on the degree of topological disorder in silicon. Note in fig. 4 that the values obtained for distances larger than $6 \AA$ seem to converge to a negative value. This is a finite-size effect, since the smallest wave vector allowed by our supercell size is $k_{\min } \sim 0.4$ $\AA^{-1}$. In the classical simulations, one finds, for both a-Si and $\mathrm{c}-\mathrm{Si}$, that $\rho(r)$ is roughly independent of temperature, and coincides with the high-temperature limit of the quantum simulations. In fact, in a classical harmonic approximation, $\rho(r)$ should be temperature-independent, as a consequence of the equipartition principle.

PI MC simulations can be used to estimate the overall anharmonicity of the vibrational modes in amorphous silicon. Even for crystalline materials, it is well known that the lattice modes are never completely harmonic, and anharmonic terms always appear to higher order when expanding in the amplitude of the lattice vibrations [21. Thus, the contribution of the anharmonic terms becomes relevant as the phonon coordinate becomes large at finite temperatures. A quantitative estimation of the overall anharmonicity of the atom vibrations can be obtained from our PI MC simulations by the ratio between kinetic and potential energy at different temperatures. According to the virial theorem, this ratio should be 1 for a harmonic solid. In fig. 5 we present the temperature dependence of this energy ratio for crystalline and amorphous silicon (open and black symbols, respectively), as derived from the quantum simulations. As expected, the anharmonicity is found to be higher for a-Si, although the kinetic-to-potential energy ratio goes to 1 (within our statistical noise) at low $T$. For the crystalline material, this energy ratio is close to 1 up to about $200 \mathrm{~K}$, and

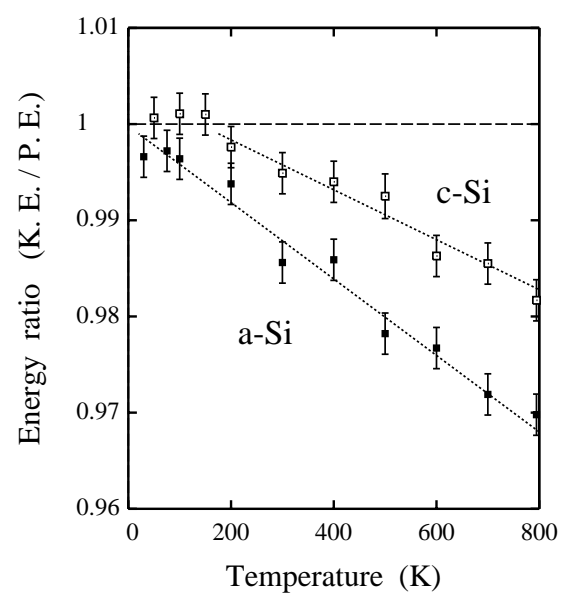

FIG. 5. Kinetic-to-potential energy ratio for crystalline (open squares) and amorphous silicon (black squares), as derived from PI MC simulations. Lines are guides to the eye. In a harmonic approximation, this energy ratio equals 1 for all temperatures (dashed line).

at higher $T$ it decreases with a slope smaller than in the case of a-Si. Note that at the scale of fig. 5 , the anharmonicity of the low-energy vibrational modes typical of amorphous materials [12,13 is not observable at low $T$. Thus, the enhanced quantum delocalization in a-Si with respect to c-Si is due (i) to a softening of the vibrational modes in the material, as found from the vibrational density of states for a-Si, even in a harmonic approximation; and (ii) to a larger anharmonicity of the vibrations in the amorphous solid, as expected from the metastability of a-Si. The resulting quantum delocalization is not negligible versus the structural disorder (described in classical terms), as seen for the RDF in figs. 1 and 2.

In summary, path-integral Monte Carlo simulations provide us with a good tool to study quantum effects in disordered materials. Quantum effects are significant for structural observables such as the radial distribution function of amorphous silicon, especially at low temperatures. Although quantitative values found by using the Stillinger-Weber potential for the interatomic interaction can change by employing other potentials, the main conclusions presented here can hardly depend on the potential employed in the simulations. Quantum simulations similar to those presented here can give information on the atom delocalization and anharmonic effects in other amorphous materials. In particular, it is expected that this method will give valuable information on hydrogenated a-Si, where the quantum delocalization of hydrogen can be crucial to understand the macroscopic properties of this material. In fact, nontrivial quantum effects have already been presented from PI MC simulations of hydrogen in c-Si [22]. Also, quantum simulations in the context of the quantum transition-state theory can give valuable information on the low-energy tunneling states that control the thermal, acoustic, and dielectric 
properties of amorphous materials at low temperatures.

This work was supported by CICYT (Spain) under Contract No. PB96-0874. Helpful discussions with R. Ramírez and J. C. Noya are acknowledged. M. A. Ramos and J. A. Vergés are thanked for critically reading the manuscript.

[1] Elliott S. R., Physics of Amorphous Materials (Longman, New York) 1990.

[2] Cusack N. E., The Physics of Structurally Disordered Matter (Adam Hilger, Bristol) 1987.

[3] Mousseau N. and Lewis L. J., Phys. Rev. Lett., 78 (1997) 1484.

[4] Barkema G. T. and Mousseau N., Phys. Rev. Lett., 77 (1996) 4358.

[5] Liu X., White B. E., Pohl R. O., Iwanizcko E., Jones K. M., Mahan A. H., Nelson B. N., Crandall R. S., and Veprek S., Phys. Rev. Lett., 78 (1997) 4418.

[6] Wooten F., Winer K., and Weaire D., Phys. Rev. Lett., 54 (1985) 1392.

[7] Kluge M. D., Ray J. R., and Rahman A., Phys. Rev. B, 36 (1987) 4234.

[8] Kluge M. D. and Ray J. R., Phys. Rev. B, 37 (1988) 4132; Luedtke W. D. and Landman U., Phys. Rev. B, 40 (1989) 1164.

[9] Biswas R., Bouchard A. M., Kamitakahara W. A., Grest G. S., and Soukoulis C. M., Phys. Rev. Lett., 22 (1988) 2280.

[10] Cook S. J. and Clancy P., Phys. Rev. B, 47 (1993) 7686.

[11] Car R. and Parrinello M., Phys. Rev. Lett., 60 (1988) 204.

[12] Buchenau U., Zhou H. M., Nucker N., Gilroy K. S., and Phillips W. A., Phys. Rev. Lett., 60 (1988) 1318; Buchenau U., J. Non-Cryst. Solids, 172-174 (1994) 384.

[13] Gil L., Ramos M. A., Bringer A., and Buchenau U., Phys. Rev. Lett., 70 (1993) 182; Ramos M. A., Gil L., Bringer A., and Buchenau U., Phys. Status Solidi A, 135 (1993) 477; Schober H. R. and Oligschleger C., Phys. Rev. B, 53 (1996) 11469.

[14] Marx, D. and Parrinello, M., Nature, 375 (1995) 216; Science, 271 (1996) 179.

[15] Feynman R. P., Statistical Mechanics (Addison-Wesley, New York) 1972.

[16] Metropolis N., Rosenbluth A. W., Rosenbluth M. N., Teller A. H., and Teller E., J. Chem. Phys., 21 (1953) 20.

[17] Gillan M. J., Phil. Mag. A, 58 (1988) 257; Ceperley D. M., Rev. Mod. Phys., 67 (1995) 279.

[18] Ramírez R. and Herrero C. P., Phys. Rev. B, 48 (1993) 14659; Herrero C. P. and Ramírez R., Phys. Rev. B, 51 (1995) 16761.

[19] Stillinger F. H. and Weber T. A., Phys. Rev. B, 31 (1985) 5262.

[20] Etherington G., Wright A. C., Wenzel J. T., Dore J. C., Clarke J. H., and Sinclair R. N., J. Non-Cryst. Solids, 48
(1982) 265; Filipponi A. and Di Cicco A., Phys. Rev. B, 51 (1995) 12322.

[21] Srivastava G. P., The Physics of Phonons (Adam Hilger, Bristol) 1990.

[22] Ramírez R. and Herrero C. P., Phys. Rev. Lett., 73 (1994) 126; Herrero C. P., Phys. Rev. B, 55 (1997) 9235. 